Full Paper

\title{
Enantioselective Detection of D- and L-Phenylalanine Using Optically Active Polyaniline
}

\author{
Edel M. Sheridan, Carmel B. Breslin* \\ Department of Chemistry, National University of Ireland Maynooth, Maynooth, Co. Kildare, Ireland \\ *e-mail: cb.breslin@may.ie
}

Received: July 15, 2004

Final version: November 1, 2004

\begin{abstract}
Chiral conducting polyaniline, which was prepared electrochemically from $(+)$ and $(-)$ camphorsulfonic acid, was utilized for the enantiomeric detection of phenylalanine in neutral phosphate buffer solution. Following electropolymerization the polymer films were dedoped in aqueous ammonia and subsequently shown to maintain their chiral character. The electrochemical window for this polymer was determined to be between $0.2 \mathrm{~V}$ and $0.8 \mathrm{~V}$ (vs. SCE) and enantiomeric discrimination between L- and D-phenylalanine was seen with the application of differential pulse voltammetry. The $(+)$ chiral polymer is shown to behave as an enantiomeric detector for the $\mathrm{D}$ enantiomer producing a peak in the voltammogram at ca. $0.7 \mathrm{~V}$ (vs. SCE) while the inverse is true for the (-) polymer, which detects the $\mathrm{L}$ enantiomer.
\end{abstract}

Keywords: Enantioselective detection, Phenylalanine, Chiral polyaniline, Differential pulse voltammetry

\section{Introduction}

In recent times there has been much interest in the production and possible use of chiral surfaces varying from single crystalline metal surfaces [1] to chiral conducting polymers [2-12]. Chiral detection is generally carried out by gas chromatography and HPLC but electrochemical detection could provide an alternative detection method and is favorable due to its simplicity, sensitivity and low cost. Various chiral conducting polymers have been developed due to their potential applications as electrodes for asymmetric electrochemical synthesis and for enantiomeric discrimination [2-12].

Much of the early work on chiral conducting polymers concentrated on the synthesis and polymerization of chiral monomers based on pyrrole bearing optically active side chains such as glucose [3], camphor [2, 4] or amino acids [5], or thiophenes with chiral groups at the 3-position $[7,8]$. More recently a facile route has been developed using an achiral monomer in the presence of a chiral inducing reagent such as a chiral acid [9-12]. This avoids the time consuming and expensive production of the chiral monomer.

The first preparation of chiral polyaniline from the achiral monomers was reported in 1994 by Wallace and co-workers $[9,11,12]$ and independently by Havinga et al. [10]. This approach involved the enantioselective electropolymerization of aniline in the presence of $(+)$ or $(-)-10$ camphorsulfonic acid (HCSA). The authors showed the polymer to be chiral using the technique of induced circular dichroism where the $(+)$ HCSA acid-doped polyaniline exhibited mirror image spectra to that of the (-) HCSA acid-doped polyaniline. These chiral polymers were also examined in a dedoped state, the $\mathrm{CSA}^{-}$anions having been removed by immersion of the polymer in aqueous ammonia, and again the spectra were found to be mirror images of each other, thus showing the polymer to be chiral even in the absence of the CSA dopant anions. It was concluded that the observed optical activity could be explained in terms of the enantiomeric $\mathrm{CSA}^{-}$anions maintaining segments of the polymer chain in a helical configuration with a preferred one-screw-sense even if removed after polymerization.

Despite extensive studies on the characterization of such chiral conducting polymers [13-16] there are very few reports on the use of these materials for enantiomeric detection of chiral molecules. Kaner and co-workers [1719] have reported that chiral polyaniline discriminates between the enantiomers of phenylalanine and derivatives of phenylalanine. This was deduced by recording the CD spectra of chiral polyaniline in the presence of $L$ and $D$ phenylalanine; an enhanced CD absorbance at $450 \mathrm{~nm}$ was observed between the chiral polymer and L-phenylalanine but not between the chiral polymer and D-phenylalanine. However, these CD measurements were made following a three-week immersion period in the amino acid solutions. Apart, from these reports, there are no other communications in the literature detailing the chiral recognition or detection properties of optically active polyaniline.

In this paper we report the chiral detection of amino acids, specifically L- and D-phenylalanine, at chiral polyaniline electrodes prepared with aniline in the presence of camphorsulfonic acid. These interactions were observed using the electrochemical technique, differential pulse voltammetry (DPV). While differential pulse voltammetry has been utilized to detect amino acids [20] and biological molecules [21] at platinum, to the best of our knowledge, this is the first report in which chiral discrimination between two enantiomers 
of amino acids at chiral polyaniline has been observed using this simple, but rapid electrochemical technique.

\section{Experimental}

Electrodes were prepared from pure platinum $(99.999+\%)$ disk, $4.0 \mathrm{~mm}$ in diameter, and optically transparent indium tin oxide coated glass (ITO) supplied by Delta Technologies with a sheet resistance of $15-25 \Omega$ (dimensions: $7 \mathrm{~mm} \times$ $50 \mathrm{~mm} \times 0.7 \mathrm{~mm}$ ). The platinum disk electrodes were set with epoxy resin in a Teflon holder and electrical contact achieved by means of a copper wire attached to the base of the metal sample with conducting silver-loaded resin. Prior to each test, the platinum working electrodes were polished to a smooth surface finish, using water lubricated 2500 grit $\mathrm{SiC}$ paper, sonicated and rinsed with distilled water. All electrochemical experiments were carried out with a threeelectrode cell configuration, using a saturated calomel reference electrode (SCE) and a platinum wire auxiliary electrode. All potentials quoted are in relation to SCE.

All chemicals were prepared using analytical grade reagents and distilled water. Aniline was distilled and stored in a dark environment under nitrogen until required. The polyaniline films were prepared using $0.3 \mathrm{~mol} \mathrm{dm} \mathrm{dm}^{-3}$ aniline

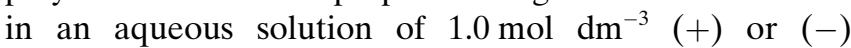
camphorsulfonic acid ( $\mathrm{pH}$ 0.9). Although camphorsulfonic acid is an organic acid, it is sufficiently soluble in water to use as an aqueous electrolyte and has been used in this capacity in a number of studies [9, 11]. Following formation, the polyaniline films were chemically dedoped by immersion in a $0.1 \mathrm{~mol} \mathrm{dm}^{-3}$ aqueous ammonia solution for a period of 1.0 hour. Any excess ammonia was removed by a second immersion for a $15-\mathrm{min}$ period in distilled water. A $0.15 \mathrm{~mol}$ $\mathrm{dm}^{-3}$ phosphate buffer solution, $\mathrm{pH} 7.5$, prepared from $\mathrm{NaH}_{2} \mathrm{PO}_{4}$ and $\mathrm{Na}_{2} \mathrm{HPO}_{4}$ was used as a supporting electrolyte in the amino acid detection experiments. The supporting electrolyte solution used in the $\mathrm{pH}$ study, consisted of a $0.15 \mathrm{~mol} \mathrm{dm}{ }^{-3}$ solution of either $\mathrm{NaH}_{2} \mathrm{PO}_{4}$ or $\mathrm{Na}_{2} \mathrm{HPO}_{4}$ adjusted to the required $\mathrm{pH}$ value using either concentrated $\mathrm{NaOH}$ or $\mathrm{H}_{3} \mathrm{PO}_{4}$. Fresh stock solutions of the $0.1 \mathrm{~mol} \mathrm{dm}{ }^{-3}$ amino acids were prepared prior to each measurement, then $0.50 \mathrm{~mL}$ of the stock solutions were added to a $20 \mathrm{~mL}$ phosphate buffer solution to give a final concentration of $2.4 \times 10^{-3} \mathrm{~mol} \mathrm{dm}^{-3}$ for the amino acid.

The polyaniline films were prepared at a constant potential of $750 \mathrm{mV}$ using a Solartron potentiostat, Model 1285. Cyclic voltammetry measurements were also carried out using the Solartron potentiostat. Differential pulse voltammetry measurements were performed on a CHI 400 EQCM, Model 400 potentiostat. The potential was scanned between $0.2 \mathrm{~V}$ and $0.80 \mathrm{~V}$ at a rate of $10 \mathrm{mV} \mathrm{s}^{-1}$. The amplitude was $50 \mathrm{mV}$, the pulse width was $0.07 \mathrm{~s}$, the sampling width was $0.03 \mathrm{~s}$ and the pulse period was $0.3 \mathrm{~s}$. Circular dichroism measurements were performed for polymers deposited at the ITO electrodes using a Jasco CD Spectrometer Model J-810 between the wavelengths of 300 and $1000 \mathrm{~nm}$.

\section{Results and Discussion}

\subsection{Preparation of Chiral Polyaniline}

Polyaniline films were electrosynthesized at platinum disk electrodes in a $1.0 \mathrm{~mol} \mathrm{dm}{ }^{-3}(+)$ or $(-)$ HSCA solution containing $0.3 \mathrm{~mol} \mathrm{dm} \mathrm{dm}^{-3}$ aniline at a constant applied potential of $0.75 \mathrm{~V}$ until a charge of $0.3 \mathrm{C} \mathrm{cm}^{-2}$ was passed. The thickness of these films was estimated as $1200 \pm 50 \mathrm{~nm}$ based on the $600 \mathrm{C} \mathrm{cm}^{-3}$ conversion factor used by Birss and co-workers $[22,23]$. This procedure, in which oxidation of polyaniline to the higher pernigraniline state is avoided, gave rise to the growth of pure polyaniline layers free from any degradation products. This was confirmed by recording the cyclic voltammogram of the prepared polyaniline films in $1.0 \mathrm{~mol} \mathrm{dm}^{-3} \mathrm{H}_{2} \mathrm{SO}_{4}$, Figure 1 . The anodic peak, observed at $140 \mathrm{mV}$, corresponds to the transition of polyaniline from the poorly conducting leucoemeraldine salt to the highly conducting emeraldine state [24] while the corresponding reduction peak is seen at $70 \mathrm{mV}$. The second redox transition is partially visible at $700 \mathrm{mV}$ and corresponds to the oxidation of polyaniline from the emeraldine to the pernigraniline state. It is clear from this voltammogram that the polyaniline formed is relatively free of any degradation products, which appear as a 'middle peak' between the leucoemeraldine/emeraldine and the emeraldine/pernigraniline states [25]. Such a peak is not observed in Figure 1.

The polyaniline films were then dedoped using a modification of the method reported by Wallace and co-workers [12]. The films were immersed in $0.1 \mathrm{~mol} \mathrm{dm}^{-3} \mathrm{NH}_{3}$ (aq) for a 1.0 hour period, removed and immersed in distilled water for a further 15 minute period. This dedoping process converts the polyaniline from the emeraldine salt to the emeraldine base, as depicted in Scheme 1.

As shown from the CD spectra presented in Figure 2, the optical activity of the polyaniline films is retained following this procedure. The polyaniline films generated from the (+)-HSCA give a large peak in the negative direction, centred at approximately $390 \mathrm{~nm}$, while a large peak,

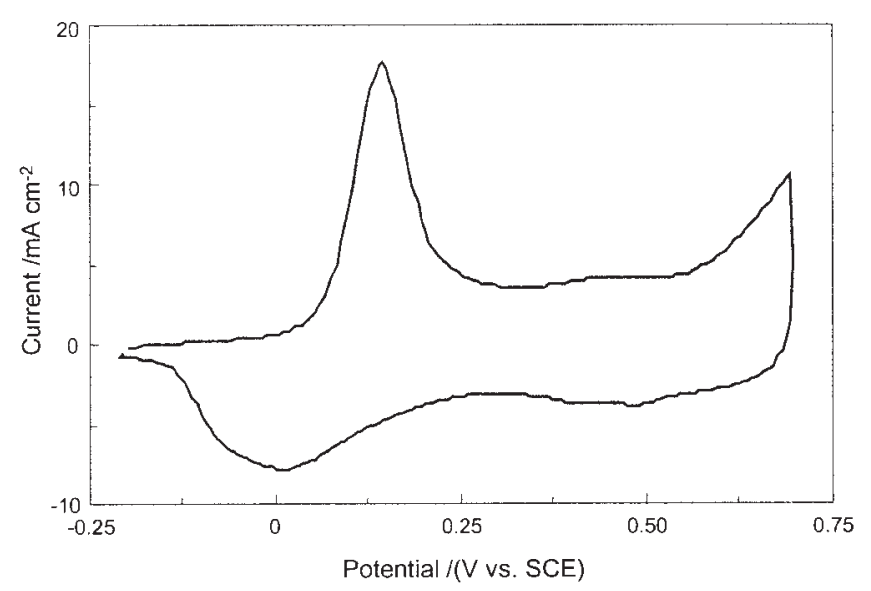

Fig. 1. Cyclic voltammogram recorded at $50 \mathrm{mV} \mathrm{s}^{-1}$ in $1.0 \mathrm{~mol}$ $\mathrm{dm}^{-3} \mathrm{H}_{2} \mathrm{SO}_{4}$ for polyaniline following formation in (+) HCSA. 


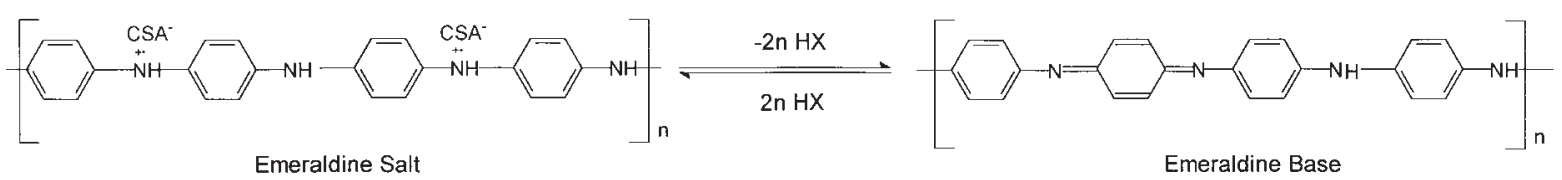

Scheme 1.

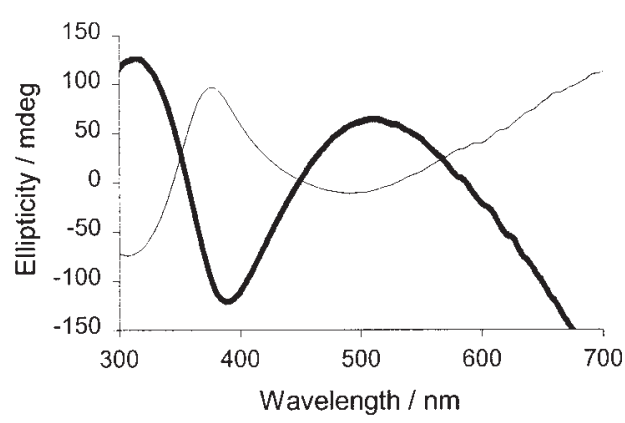

Fig. 2. CD spectra of dedoped polyaniline electropolymerized in $(-)(-)$ HCSA, in $(-)(+)$ HCSA on ITO, de-doped by a 1.0 hour immersion in $0.1 \mathrm{~mol} \mathrm{dm}^{-3}$ aqueous ammonia.

centred at $390 \mathrm{~nm}$, in the positive direction is measured for the film prepared from the (-) HSCA. Weaker mirror-like absorptions are seen at about $500 \mathrm{~nm}$. These spectra are similar to those presented previously for chiral polyaniline in the emeraldine base state [14]. This behavior has been attributed to the conformational properties of the polymer backbone induced by the chiral $\mathrm{CSA}^{-}$dopants $[8,10]$. As shown here and in other communications [19], the helical chain conformations do not relax when the dopant is removed.

\subsection{Electrochemical Detection Measurements}

There was no evidence of any interaction between the phenylalanine and the chiral polyaniline from cyclic voltammetry measurements. Typical data are shown in Figure 3 for the cyclic voltammetry of the chiral polymer at a scan rate of $200 \mathrm{mV} \mathrm{s}^{-1}$ in a phosphate buffer solution at $\mathrm{pH} 7.5$ with $2.4 \mathrm{mmol} \mathrm{dm}^{-3}$ of D- and L-phenylalanine. As evident from these data, there is no indication of any interaction between the chiral polyaniline and the amino acid, or of any chiral discrimination.

However, it is seen clearly from the data recorded using differential pulse voltammetry, presented in Figures 4 and 5, that the chiral polyaniline exhibits molecular recognition properties. The plot presented in Figure 4 was recorded with the dedoped polymer in an amino acid-free phosphate buffer solution polarized between 0.2 and $0.8 \mathrm{~V}$. The average current recorded within this potential region is approximately $12 \mu \mathrm{A} \mathrm{cm}^{-2}$, indicating little electrochemical activity. This is consistent with the electrochemical activity of polyaniline in a near-neutral solution. It is well documented in the literature that polyaniline appears to become less electroactive with increasing $\mathrm{pH}$ [26].

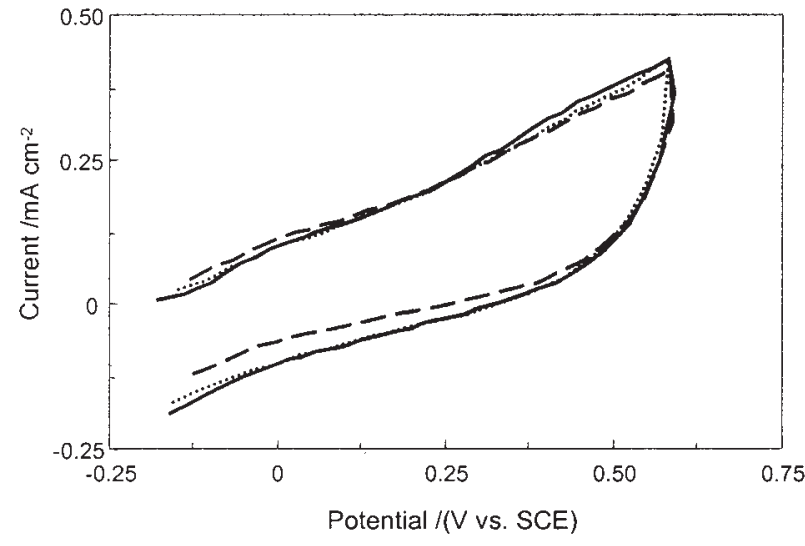

Fig. 3. Cyclic Voltammograms recorded at $200 \mathrm{mV} \mathrm{s}^{-1}$ for dedoped $(+)$ polyaniline cycled in $(-) 0.15 \mathrm{~mol} \mathrm{dm}^{-3}$ phosphate buffer at $\mathrm{pH} 7.5,(\cdots \cdots)$ in phosphate buffer solution with $2.4 \mathrm{mmol} \mathrm{dm}^{-3} \mathrm{~L}$-phenylalanine, (--) in phosphate buffer solution with $2.4 \mathrm{mmol} \mathrm{dm}^{-3}$ D-phenylalanine.

Once this electrochemical window was determined, this potential range was chosen for all enantiomeric detection experiments. These background differential pulse voltammograms were recorded in the phosphate buffer solution prior to the addition of phenylalanine. On the addition of phenylalanine very different behavior was observed, as shown in Figure 5. In this figure, four differential pulse voltammograms are presented; polarization of the (+) polyaniline in the phosphate buffer solution containing either the D- or L-phenylalanine and polarization of the $(-)$ polyaniline in the presence of the $\mathrm{D}$ - or L-phenylalanine. As can be seen no significant response occurs when the $(+)$ chiral polyaniline is exposed to the $\mathrm{L}$ - enantiomer of the phenylalanine but a clearly defined peak at ca. $0.7 \mathrm{~V}$ is

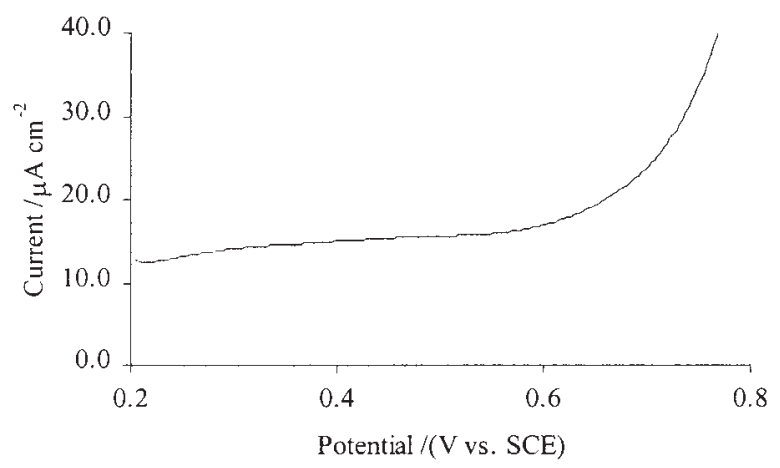

Fig. 4. Differential pulse voltammogram recorded for dedoped (+) polyaniline in a $0.15 \mathrm{~mol} \mathrm{dm}{ }^{-3}$ phosphate buffer at $\mathrm{pH} 7.5$.

(C) 2005 WILEY-VCH Verlag GmbH \& Co. KGaA, Weinheim 
(a)

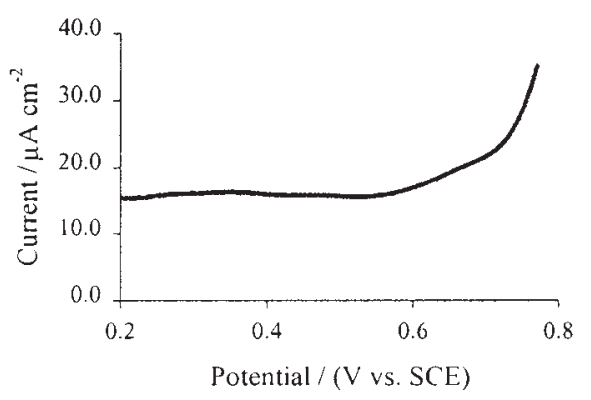

(c)

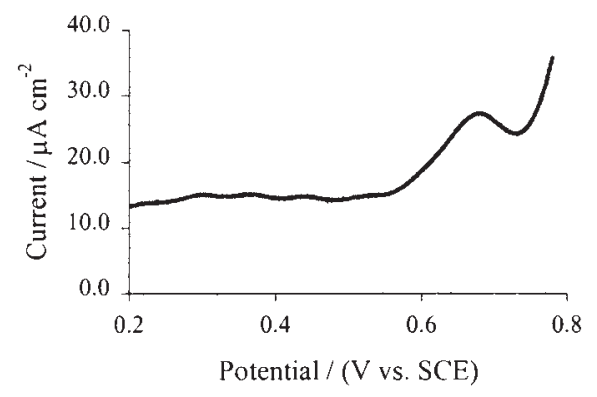

(b)

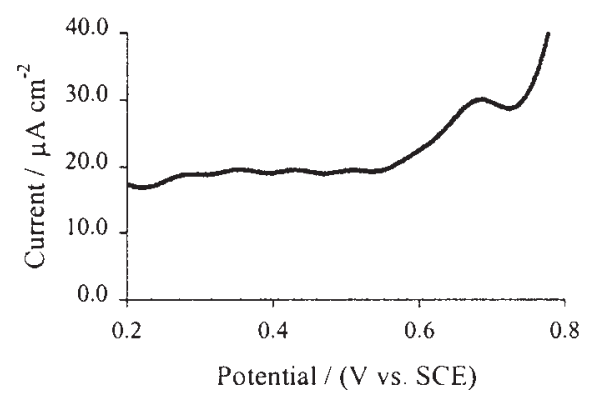

(d)

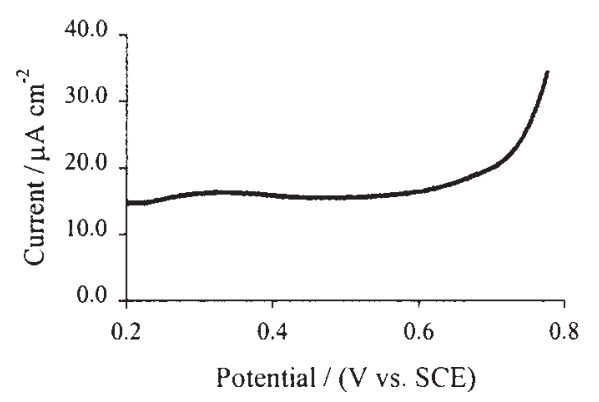

Fig. 5. Differential pulse voltammograms recorded for dedoped chiral polyaniline in a $0.15 \mathrm{~mol} \mathrm{dm}^{-3}$ phosphate buffer solution at pH 7.5 with $2.4 \mathrm{mmol} \mathrm{dm}^{-3}$ phenylalanine; a) (+) polyaniline with L-phenylalanine, b) (-) polyaniline with L-phenylalanine, c) (+) polyaniline with D-phenylalanine, d) (-) polyaniline with D-phenylalanine.

observed when the $(+)$ polyaniline is polarized in the presence of the D-phenylalanine. The inverse behavior is observed with the (-) polyaniline; detection of the Lenantiomer, but no detection of the D-enantiomer. These data show clearly that the polymer possesses chiral recognition properties. Nevertheless, it can be seen, particularly in Figure 5(a), that there is some evidence of a signal, although considerably lower in intensity, for the $(+)$ polyaniline and $\mathrm{L}$-phenylalanine. However, this is consistent with a number of previous studies on the electrochemical oxidation of Dglucose at chiral platinum surfaces [27, 28]. In these studies, oxidation of both enantiomers was observed, but the rate of oxidation was always greater with one of the enantiomers giving rise to electrochemical enantioselectivity.

In order to gain some understanding of the mechanism of this interaction, differential pulse voltammograms were recorded for the $(+)$ and $(-)$ polyaniline in the presence of D- and L-phenylalanine, as a function of the phenylalanine concentration and at different $\mathrm{pH}$ values. In Figure 6, the peak current is shown as a function of the analyte concentration for $(+)$ polyaniline cycled in the presence of $\mathrm{D}$ phenylalanine. A linear response is observed with phenylalanine concentrations below $1.0 \mathrm{mmol} \mathrm{dm}{ }^{-3}$. However, with increasing concentrations a saturation effect is observed. Similar results were observed on recording the

Electroanalysis 2005, 17, No. 5-6

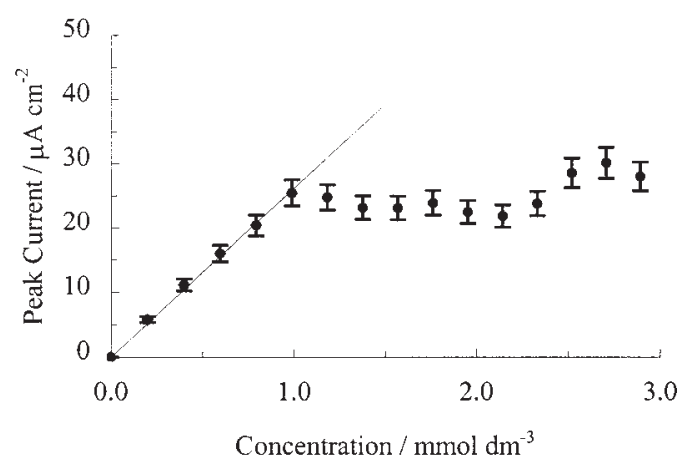

Fig. 6. Plot of peak current recorded with $(+)$ polyaniline as a function of the concentration of D-phenylalanine.

differential pulse voltammograms of $(-)$ polyaniline in a solution of L-phenylalanine.

In Figure 7, data are presented to illustrate the influence of repeated cycling on this chiral response. In this plot, the peak current is presented as a function of the cycle number for the repeated cycling of $(+)$ polyaniline in a $2.4 \mathrm{mmol}$ $\mathrm{dm}^{-3}$ solution of D-phenylalanine in phosphate buffer. A significant decrease in the peak current is observed with the first 10 cycles and then the rate of decay becomes smaller to (C) 2005 WILEY-VCH Verlag GmbH \& Co. KGaA, Weinheim 


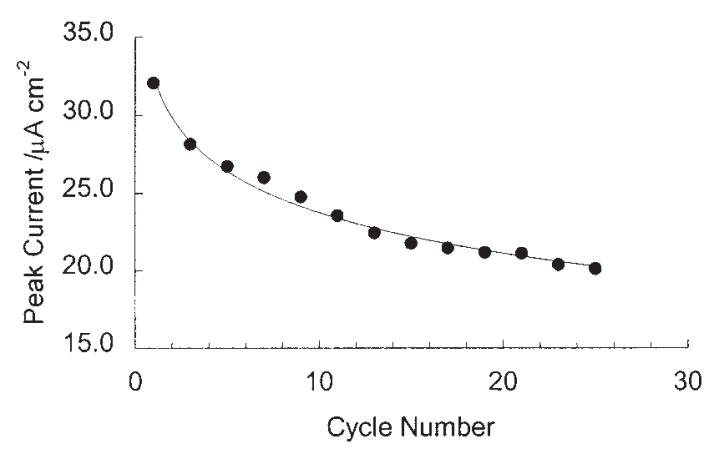

Fig. 7. Plot of peak current recorded with $(+)$ polyaniline as a function of the cycle number in a phosphate buffer solution with $2.4 \mathrm{mmol} \mathrm{dm}^{-3}$ D-phenylalanine.

reach a near steady-state condition. This may suggest that the surface becomes saturated with the phenylalanine.

For the $\mathrm{pH}$ studies, the experiments were performed in a non-buffered phosphate solution. At $\mathrm{pH}$ values between 7.0 and 9.0 clear chiral detection properties were observed; i.e. detection of the D-phenylalanine with the $(+)$ polyaniline and detection of the L-phenylalanine with the $(-)$ polyaniline. However, as the $\mathrm{pH}$ was lowered below 7.0, the signal decayed. The isoelectronic point of phenylalanine is 5.75 . Consequently, as the $\mathrm{pH}$ of the solution is increased to values in the region of 7.0 and then higher, the concentration of the zwitterionic form increases with dissociation of the carboxylic acid group to give the carboxylate group. There is much evidence in the literature to suggest that the oxidation of amino acids at platinum and gold occurs by the adsorption of the amino acid through the carboxylate group, followed by decarboxylation at more positive potentials [29]. Interestingly, decarboxylation of phenylalanine has been reported to occur at potentials in the vicinity of $0.30 \mathrm{~V}$ (SCE) at $\mathrm{Au}(111)$ electrodes [1]. Also, Moreno et al. [20] observed peaks in the differential pulse voltammograms recorded for cysteine, tryptophan and tyrosine in the potential vicinity of 0.6 to $0.8 \mathrm{~V}(\mathrm{Ag} \mid \mathrm{AgCl})$ at a polycrystalline platinum electrode. Consequently, the peak observed at approximately $0.7 \mathrm{~V}$, in Figure 5 , may be connected with the oxidation of the amino acid at the platinum substrate, with the polymer acting as a chiral membrane. Indeed, on recording the differential pulse voltammograms of platinum in the phenylalanine-containing phosphate buffer solution an oxidation wave was observed with a peak potential of $0.64 \mathrm{~V}(\mathrm{SCE})$. Furthermore, on increasing the thickness of the polymer, the peak current decayed. For example, when the electropolymerization charge was $0.3 \mathrm{C} \mathrm{cm}^{-2}$, the peak current density was $30 \pm 2.0 \mu \mathrm{A} \mathrm{cm}^{-2}$, but at a charge of $0.8 \mathrm{C} \mathrm{cm}^{-2}$ the peak current density was reduced to $23 \pm$ $2.0 \mu \mathrm{A} \mathrm{cm}^{-2}$

Work is ongoing to determine the exact origin of this peak. However, it is very clear that the chiral polyaniline possesses chiral recognition properties, being able to discriminate between the D- and L-enantiomers of phenylalanine.

\section{Conclusions}

Chiral polyaniline was prepared from $(+)$ and $(-)$ camphorsulfonic acid. The polymer remained chiral on removing the CSA dopant anions. Rapid and efficient enantiomeric detection of $\mathrm{L}$ - and D-phenylalanine was observed at the chiral polymer using differential pulse voltammetry. A clear oxidation peak was observed at approximately $0.7 \mathrm{~V}$ on polarizing $(+)$ polyaniline in the presence of D-phenylalanine, but no significant peak was observed on polarizing the polymer in the presence of $\mathrm{L}$ - phenylalanine.

\section{Acknowledgements}

The authors gratefully acknowledge the support of this work by Enterprise Ireland under the Basic Science Research Grant Programme and also would like to thank Professor John Kelly, Trinity College Dublin for the CD measurements.

\section{References}

[1] H. Q. Li, A. C. Chen, S. G. Roscoe, J. Lipkowski, J. Electroanal. Chem. 2001, 500, 299.

[2] M. Salmon, G. Bidan, J. Electrochem. Soc. 1985, 132, 1897.

[3] J. C. Moutet, E. Saint-Aman, F. Tran-Van, P. Angibeaud, J. P. Utille, Adv. Mater. 1992, 4, 511.

[4] M. Salmon, M. Saloma, G. Bidan, E. M. Genies, Electrochim. Acta 1989, 34, 117.

[5] J. Grimshaw, S. D. Perera, J. Chem. Soc., Perkin Trans. 2 1989, 1711

[6] S. Pleus, M. Schwientek, Synth. Commun. 1997, 27, 2917.

[7] D. Kotkar, V. Joshi, P. K. Ghosh, J. Chem. Soc., Chem. Commun. 1988, 917.

[8] M. Lemaire, D. Delabouglise, R. Garreau, A. Guy, J. Roncali, J. Chem. Soc., Chem. Commun. 1988, 658.

[9] M. R. Majidi, L. A. P. Kane-Maguire, G. G. Wallace, Polymer 1994, 35, 3113

[10] E. E. Havinga, M. M. Bouman, E. W. Meijer, A. Pomp, M. M. J. Simenon, Synth. Met. 1994, 66, 93.

[11] M. R. Majidi, L. A. P. Kane-Maguire, G. G. Wallace, Polymer 1995, 36, 3597.

[12] M. R. Majidi, L. A. P. Kane-Maguire, G. G. Wallace, Polymer 1996, 37, 359.

[13] L. A.P. Kane-Maguire, I. D. Norris, G. G. Wallace, Synth. Met. 1999, 101, 817

[14] V. Egan, R. Bernstein, L. Hohmann, T. Tran, R. B. Kaner, Chem. Commun. 2001, 801.

[15] S. A. Ashraf, L. A. P. Kane-Maguire, M. R. Majidi, S. G. Pyne, G. G. Wallace, Polymer 1997, 38, 2627.

[16] M. R. Majidi, L. A. P. Kane-Maguire, G. G. Wallace, Aust. J. Chem. 1998, 51, 23.

[17] H. L. Guo, C. M. Knobler, R. B. Kaner, Synth. Met. 1999, $101,44$.

[18] J. X. Huang, V. M. Egan, H. L. Guo, J. Y. Yoon, A. L. Briseno, I. E. Rauda, R. L. Garrell, C. M. Knobler, F. M. Zhou, R. B. Kaner, Adv. Mater., 2003, 15, 1158.

[19] R. B. Kaner, Synth. Met. 2001, 125, 65.

[20] L. Moreno, A. Merkoci, S. Alegret, S. Hernandez-Cassou, J. Saurina, Anal. Chim. Acta 2004, 507, 247.

[21] G. Erdogdu, A. E. Karagozler, Talanta 1997, 44, 2011.

(C) 2005 WILEY-VCH Verlag GmbH \& Co. KGaA, Weinheim 
[22] H. N. Dinh, P. Vanysek, V. I. Birss, J. Electrochem. Soc. 1999, $146,3324$.

[23] H. N. Dinh, V. I. Birss, J. Electrochem. Soc. 2000, 147, 3775

[24] C. C. Hu, E. Chen, J. Y. Lin, Electrochim. Acta 2002, 47, 2741.

[25] W. C. Chen, T. C. Wen, A. Gopalan, Electrochim. Acta 2002, $47,4195$.
[26] L. Nyholm, L. M. Peter, J. Chem. Soc., Faraday T. 1994, 90, 149.

[27] G. A. Attard, J. Phys. Chem. B 2001, 105, 3158.

[28] G. A. Attard, C. Harris, E. Herrero, J. Feliu, Faraday Discuss. 2002, 121, 253.

[29] J. E. I. Wright, K. Fatih, C. L. Brosseau, S. Omanovic, S. G. Roscoe, J. Electroanal. Chem. 2003, 550, 41. 